\title{
Studies of Polyelectrolyte Solutions IV. Effects of Ionic Strength on the Effective Polyion Charge
}

\author{
Eckhard NORDMEIER \\ Department of Physical Chemistry, University of Osnabrück, \\ 4500 Osnabrück, FRG
}

(Received March 5, 1992)

\begin{abstract}
Quasi-Elastic light scattering experiments have been performed on aqueous solutions of sodium poly(styrene sulfonate) $\left(M_{w}=3.5 \times 10^{5} \mathrm{~g} \mathrm{~mol}^{-1}\right)$ in the ionic strength range of 0.005 to $2 \mathrm{M}$ at $T=20^{\circ} \mathrm{C}$. The angular dependence of the apparent diffusion coefficient was analyzed within the framework of the Fujime-Maeda theory for semiflexible rods. At zero polyion concentration both the overall translational and the rotational diffusion coefficient increase with the ionic strength, while the difference between the translational diffusion coefficients perpendicular and parallel to the rod axis decreases. The molecular structure of a PSS-polyion is also quite well described by the model of a wormlike prolate ellipsoid, where the ratio of the minor to the major semiaxis rises from 0.29 at low ionic strength $(I=0.005 \mathrm{M})$ to 1 at the $\theta$-state. By solving the Poisson-Boltzmann equation we have determined the effective polyion charge, $Z_{\text {eff }}$, that is not neutralized by the small ions at a distance $r=d_{\mathrm{h}} / 2$ from the PSS-skeleton, where $d_{\mathrm{h}}$ is the hydrodynamic diameter of the polyion. It is found: (1) $Z_{\text {eff }}$ is independent of the ionic strength; and (2) $Z_{\text {eff }}$ is much smaller than the corresponding $Z$-values obtained from dialysis equilibrium experiments. The reason for this discrepancy is clear. The dialysis technique yields the surface charge of the polyion, while transport techniques such as diffusion experiments yield the effective charge, $Z_{\text {eff }}$. The latter incorporate hydrodynamic and electrostatic coupling forces acting between the polyion and the associated ion cloud, while the former do not.

KEY WORDS Quasi-Elastic Light Scattering / Poly(styrene sulfonate) / Translational Diffusion Coefficient / Hydrodynamic Radius / Effective Polyion Charge /
\end{abstract}

We have recently ${ }^{1-3}$ investigated the physicochemical properties of sodium poly(styrene sulfonate) $\left(M_{w}=3.5 \times 10^{5} \mathrm{~g} \mathrm{~mol}^{-1}\right)$ by static light scattering and viscosity measurements. In addition to the determination of the intensity of the scattered light, from which we derived the radius of gyration, $\left\langle S^{2}\right\rangle_{z}^{1 / 2}$, and the second virial coefficient, $A_{2}$, we determined the net polyion charge, $Z_{\text {net }}$, by the dialysis equilibrium technique over a wide range of $\mathrm{NaNO}_{3}$ concentrations. The analysis of the data from these measurements has indicated that a PSS-polyion can be described well as a semiflexible rod or a wormlike coil.

In the present work, we study the dynamical behavior of PSS-polyions by means of quasi- elastic light scattering (QELS). The Brownian movement of these polyions is a superposition of translational, rotational, and flexing motions. It should be noted that the translation of a semiflexible rod is anisotropic in solution; the sideway diffusion coefficient, $D_{1}$, is smaller than the lengthway diffusion coefficient, $D_{3}$. As a consequence the translational diffusion of the chain is no longer independent of the rotational diffusion.

The plan of the present paper is as follows. We will at first review the Maeda-Fujime ${ }^{4}$ relation for the apparent diffusion coefficient, $D_{\text {app }}(q, c)$. We apply this in a slightly modified form to the wormlike chain and compute the adjustable parameters $d_{\mathrm{h}}, L_{\mathrm{h}}$, and $D_{3}-D_{1} . d_{\mathrm{h}}$ 
is the hydrodynamic diameter and $L_{\mathrm{h}}$ the length of the wormlike chain. Attention is confined to low scattering vectors, where the relevant and interesting observations are to be found. Second, we will calculate the fraction, $F(r)$, of polyion charge that is neutralized by the counterions $\left(\mathrm{Na}^{+}\right)$within the polyion cylinder with the radius $r=d_{\mathrm{h}} / 2$. Therefore, we solve numerically the Poisson-Boltzmann equation. The results will be used to estimate the effective polyion charge, $Z_{\text {eff }}$. Third, we compute the hydrodynamic radius, $R_{\mathrm{h}}$, by means of Schurr's ${ }^{5}$ equation for the diffusion of a charged, permeable gel bead. This equation takes into account both solvent and electrolyte dissipation, while the EinsteinStokes relation for $R_{\mathrm{h}}$ accounts only solvent dissipation. The data of $R_{\mathrm{h}}$ are then combined with those of $\left\langle S^{2}\right\rangle_{z}^{1 / 2}$ and analyzed with regard to the model of a prolate ellipsoid. Fourth, we study the polyion and added salt concentration dependence of the translational diffusion coefficient, $D_{\mathrm{z}}$, by means of the theories derived by Pyun-Fixman ${ }^{6}$ and Schurr ${ }^{7}$. Finally, we will discuss both the usefulness and the weakness of our treatments.

\section{EXPERIMENTAL}

\section{Materials}

Sodium poly(styrene sulfonate) (NaPSS) was purchased from Pfannenschmidt, FRG. The molar mass was $3.5 \times 10^{5} \mathrm{~g} \mathrm{~mol}^{-1}$ and the degree of sulfonation $84 \%$. The nonuniformity, $U$, was given by $U<0.12$. Stock solutions were prepared by dissolving the polymer in a proper concentration of $\mathrm{NaNO}_{3}$ and then dialyzing against aqueous solutions of the same salt concentration for at least 5 days in a special cell at constant volume. The dilutions were made dust-free by centrifugation at $10000 \mathrm{rpm}$ for $1-3 \mathrm{~h}$ and subsequent filtration through $0.22-\mu \mathrm{m}$ Millipore, sterilized, one-way filters. For each ionic strength $(0.005 \leq I \leq 2 \mathrm{M})$ six different polyion concentrations, $c_{\mathrm{p}}$, have been studied in the range of $0.2 \times 10^{-3}$ to $1.2 \times 10^{-3} \mathrm{~g} \mathrm{~cm}^{-3}$, where $c_{\mathrm{p}}$ was determined spectrophotometrically at $261 \mathrm{~nm}$ after filtration. $\mathrm{NaNO}_{3}$ (Merck) was used without further purification.

\section{Quasi-Elastic Light Scattering}

The light scattering measurements were carried out in the angular range $10-150^{\circ}$ at $20^{\circ} \mathrm{C}$ with the red line $\left(\lambda_{0}=632.8 \mathrm{~nm}\right)$ of a He-Ne laser (Spectra Physics, Model 107S/207). Details of the instrument are given in ref 8 . The data analysis was performed with a cumulant ${ }^{9}$ expansion method up to the third order. A linearized expansion formula was found not be very good for the normalized autocorrelation function, $g(\tau)$, at intermediate and large $q^{2}$-values. So, we adopt

$$
\begin{aligned}
\ln [|g(\tau)|]=- & \bar{\Gamma} \tau+\frac{\mu_{2}}{2 \bar{\Gamma}^{2}}(\bar{\Gamma} \tau)^{2} \\
& -\frac{\mu_{3}}{6 \bar{\Gamma}^{3}}(\bar{\Gamma} \tau)^{3}+\cdots
\end{aligned}
$$

where $\bar{\Gamma}$ is the average first cumulant which is related to the apparent diffusion coefficient $D_{\text {app }}$ by

$$
\bar{\Gamma}=D_{\text {app }} q^{2} .
$$

$q$ is the scattering vector, and $\mu_{2} / \bar{\Gamma}^{2}$ characterizes the width of the distribution, $G(\Gamma)$, of the first cumulants. $\tau$ is the correlation time.

\section{RESULTS AND DISCUSSION}

\section{$\Delta t$-Dependence of $D_{\text {app }}$}

Figure 1 shows plots of the apparent diffusion coefficient, $D_{\text {app }}$, versus the sample time, $\Delta t$, for NaPSS at various ionic strengths. The polyion concentration, $c_{\mathrm{p}}$, is $0.2 \times 10^{-3} \mathrm{~g} \mathrm{~cm}^{-3}$, the scattering angle is $30^{\circ}$, and the temperature is $20^{\circ} \mathrm{C}$. For small $\Delta t(\Delta t<20 \mu \mathrm{s}), D_{\text {app }}$ increases nearly exponential as $\Delta t$ goes to zero, while $D_{\text {app }}$ is almost independent of $\Delta t$ as $\Delta t$ becomes large. In the treatment that follows all parameters are related to zero 


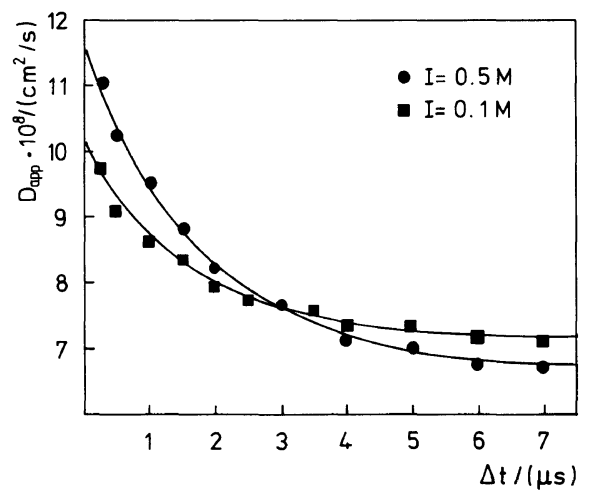

Figure 1. Dependence of $D_{\text {app }}$ on the sampling time, $\Delta t$, for $c_{\mathrm{p}}=0.2 \mathrm{~g}^{-1} \mathrm{NaPSS}$ at $\theta=30^{\circ}$ and $T=20^{\circ} \mathrm{C}$. $I=0.1$ (a) and $0.5 \mathrm{M}(\bigcirc)$.

correlation time, $\tau$. We therefore have extrapolated the $D_{\text {app }}$-data to $\Delta t=0$. An appropriate fit-formula is

$$
D_{\text {app }}=a+b[\exp (-c \times \Delta t)],
$$

where $a, b$, and $c$ are adjustable parameters $\left(D_{\text {app }}(\Delta t=0)=a+b\right)$.

\section{Angular Dependence of $D_{\mathrm{app}}$}

By definition of the first cumulant, ${ }^{9} \bar{\Gamma}=$ $-\lim _{\tau \rightarrow 0} \mathrm{~d}(\ln |g(\tau)|) / \mathrm{d} \tau$, where the limit value is taken at $\tau=0$ as pointed out above. Fujime and $\mathrm{Maeda}^{4}$ have derived an expression of the apparent diffusion coefficient, $D_{\text {app }}$, for a semiflexible rod, that is

$$
\begin{aligned}
D_{\text {app }}= & D_{0}+\left(L^{2} / 12\right) \theta f_{1}\left(k, l_{\mathrm{p}}\right) \\
& +\left(D_{3}-D_{1}\right)\left[f_{2}\left(k, l_{\mathrm{p}}\right)-1 / 3\right] \\
& +\sum_{m=2}^{\infty} D_{\mathrm{m}} \times a_{\mathrm{m}}\left(k, l_{\mathrm{p}}\right) .
\end{aligned}
$$

Here, $D_{0}=\left(2 D_{1}+D_{3}\right) / 3$ is the overall translational diffusion coefficient, $D_{1}$ and $D_{3}$ are, respectively, the translational diffusion coefficients perpendicular and parallel to the mean axis of the rod, $\theta$ is the end-overend rotational diffusion coefficient, and $L$ is the contour length of the semiflexible rod. $D_{\mathrm{m}}=\left(k_{\mathrm{B}} \times T / 4 \pi \eta L\right)\left(1+f_{\mathrm{m}}\right)$ is the $m$-th bending mode, where an explicit formula of the term $f_{\mathrm{m}}$ is found elsewhere. ${ }^{10}$ For a rigid rod $f_{\mathrm{m}}=-1$ and $D_{\mathrm{m}}$ becomes zero for all $\mathrm{m}$. The functions $f_{1}\left(k, l_{\mathrm{p}}\right), f_{2}\left(k, l_{\mathrm{p}}\right)$, and $a_{\mathrm{m}}\left(k, l_{\mathrm{p}}\right)$ depend both on the parameter $k=q \times L / 2$ and the persistence length, $l_{\mathrm{p}}$, where $q$ is the wave vector. Their explicit forms are found elsewhere. ${ }^{11,12}$ At $k=0, f_{1}, f_{2}$, and $a_{\mathrm{m}}$ are all zero, and $f_{1}=1, f_{2}=1 / 3$, and $a_{\mathrm{m}}=1$ as $k \rightarrow \infty$.

Equation 4 is quantitatively correct only for cases of very thin and long rods, ${ }^{11}$ i.e., for $d \rightarrow 0$ and $q \times L \gg 1$. Here, $d$ is the geometrical diameter of the rod. For practical values of $d(d=0.3-2.0 \mathrm{~nm})$ that are of our interest eq 4 must be modified. Previously, ${ }^{2}$ we have shown that a PSS-polyion is quite well described by the model of a wormlike coil. It is therefore reasonable to substitute $D_{0}$ and $\theta$ in eq 4 by the relationships derived for a wormlike coil.

One of the most commonly used set of expressions are those obtained by Hearst. ${ }^{13}$ At the limit of small $l_{\mathrm{p}}\left(L / 2 l_{\mathrm{p}} \gg 1\right)$ it holds

$$
\begin{gathered}
D_{0}=\frac{k_{\mathrm{B}} T}{3 \pi \eta_{0} L}\left[1.843\left(L / 2 l_{\mathrm{p}}\right)^{0.5}\right. \\
\left.-\ln \left(a / 2 l_{\mathrm{p}}\right)-2.431-\frac{a}{d_{\mathrm{h}}}\right] \\
\theta=\frac{k_{\mathrm{B}} T}{6 \pi L^{2} l_{\mathrm{p}}}\left[0.716\left(L / 2 l_{\mathrm{p}}\right)^{0.5}\right. \\
\left.-0.636 \ln \left(a / 2 l_{\mathrm{p}}\right)-1.548+0.64 \frac{a}{d_{\mathrm{h}}}\right],
\end{gathered}
$$

where $l_{\mathrm{p}}$ is the persistence length, $a$ the monomer length and $d_{\mathrm{h}}$ the hydrodynamic diameter. But we have also to account that a PSS-polyion changes its hydrodynamic dimension and shape when the ionic strength, $I$, in the solution is varied. We therefore replace $L$ by $L_{\mathrm{h}}$ in the term $\left(L^{2} / 12\right)$ of eq 4 , where $L_{\mathrm{h}}$ is the hydrodynamic length of the rod, as proposed by Fujime and Maeda. ${ }^{14}$ Additionally, we neglect the contribution of the term $\sum_{m} a_{\mathrm{m}}\left(k, l_{\mathrm{p}}\right) D_{\mathrm{m}}$ to $D_{\mathrm{app}}$. This is allowed because (1) it is very difficult to discuss quantitatively the size of $a_{\mathrm{m}}$, and (2) flexing 
E. NORDMEIER

Table I. Diffusion coefficients and hydrodynamic lengths of PSS in aqueous solutions of $\mathrm{NaNO}_{3}$ at $20^{\circ} \mathrm{C}$

\begin{tabular}{lccccccccc}
\hline$I / \mathrm{M}$ & $\begin{array}{c}D_{\mathrm{O}} \times 10^{8} / \\
\mathrm{cm}^{2} \mathrm{~s}^{-1}\end{array}$ & $\theta / \mathrm{s}^{-1}$ & $\theta_{\mathrm{E}} / \mathrm{s}^{-1}$ & $\begin{array}{c}D_{1} \times 10^{8 \mathrm{a}} / \\
\mathrm{cm}^{2} \mathrm{~s}^{-1}\end{array}$ & $\begin{array}{c}D_{3} \times 10^{8 \mathrm{~b}} / \\
\mathrm{cm}^{2} \mathrm{~s}^{-1}\end{array}$ & $\begin{array}{c}\Delta \times 10^{8} / \\
\mathrm{cm}^{2} \mathrm{~s}^{-1}\end{array}$ & $d_{\mathrm{h}} / \mathrm{nm}$ & $L_{\mathrm{h}} / \mathrm{nm}$ & $l_{\mathrm{p}} / \mathrm{nm}$ \\
\hline 0.005 & 8.6 & 763 & 721 & 7.5 & 10.7 & 3.2 & 13.1 & 216 & 16.3 \\
0.01 & 8.9 & 905 & 844 & 7.9 & 11.0 & 3.1 & 11.5 & 196 & 14.1 \\
0.02 & 9.2 & 1017 & 958 & 8.2 & 11.1 & 2.9 & 10.3 & 184 & 12.8 \\
0.05 & 9.5 & 1158 & 1093 & 8.5 & 11.3 & 2.8 & 8.4 & 171 & 11.5 \\
0.10 & 9.9 & 1410 & 1319 & 9.0 & 11.7 & 2.7 & 6.2 & 154 & 9.8 \\
0.20 & 10.1 & 1506 & 1525 & 9.2 & 11.8 & 2.6 & 5.4 & 133 & 9.3 \\
0.50 & 10.9 & 2008 & 1981 & 10.1 & 12.5 & 2.4 & 4.7 & 125 & 7.4 \\
1.00 & 11.8 & 2632 & 2576 & 11.0 & 13.2 & 2.2 & 3.8 & 110 & 6.0 \\
2.00 & 12.4 & 3174 & 3171 & 11.8 & 13.6 & 1.9 & 3.5 & 98 & 5.2 \\
\hline Error \% & $2-5$ & $5-10$ & 10 & $2-5$ & $2-5$ & 5 & $10-15$ & 10 & $10-15$ \\
\hline
\end{tabular}

a $D_{1}=\left(3 D_{\mathrm{o}}-\Delta\right) / 3$.

b $\Delta=D_{3}-D_{1}$.

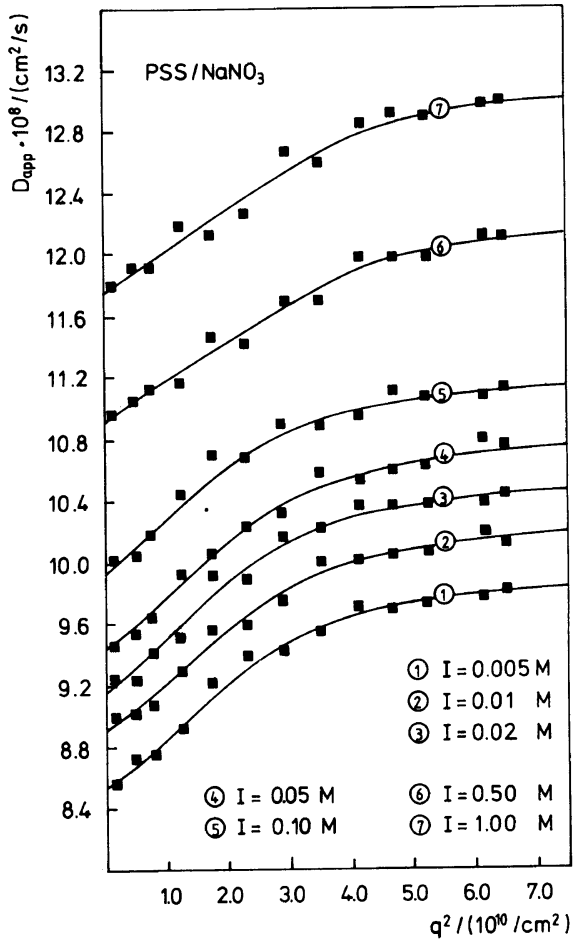

Figure 2. $D_{\text {app }}$ versus $q^{2}$ relationships of NaPSS for various ionic strengths, $I$, at $c_{\mathrm{p}}=0 \mathrm{~g}^{-1}$ and $T=20^{\circ} \mathrm{C}$. The solid lines denote the semiflexible rod values due to eq 4 .

motions become only important at high $q$ that are not inspected here.

Thus, the only unknown parameters of eq 4 are $d_{\mathrm{h}}, L_{\mathrm{h}}$, and the anisotropy, $\Delta=D_{3}-D_{1}$. We therefore consider these parameters as adjustable and compute them by the method of least squares. Using the parameter set $L=470 \mathrm{~nm}, a=0.25 \mathrm{~nm}$, and $\eta$ of $\mathrm{NaNO}_{3}$ (aq) at $T=20^{\circ} \mathrm{C}$, we then obtain the diffusion coefficients and lengths listed in Table I. The persistence lengths, given in column (10), are from ref 2.

Figure 2 shows plots of $D_{\text {app }}$ at $c_{\mathrm{p}}=0$ versus $q^{2}$ for different ionic strengths, $I$. The theoretical data are dissigned by solid lines and the experimental by squares. It is seen that most of the experimental points lie very close to the theoretical curves, suggesting that the experimental spectra for any $I$ studied so far can be fitted quite well by adjusting $d_{\mathrm{h}}, L_{\mathrm{h}}$, and $\Delta$ appropriate to each $I$. From Table $\mathrm{I}$ it is seen that $d_{\mathrm{h}}, L_{\mathrm{h}}$, and $\Delta$ decrease with increasing $I$, while simultaneously $D_{0}$ and $\theta$ increase. The overall translational diffusion coefficient, $D_{0}$, is different from those of $D_{1}$ and $D_{3} ; D_{3}$ is larger than $D_{1}$, suggesting that the translational diffusion parallel to the mean axis of the "wormlike rod" is the preferential one. The reasons for these trends will become clear in the following.

At this point, however, we should discuss the term hydrodynamic in some detail. Poly- 
ions such as PSS possess ionic groups, which, in a polar solvent, will be associated with salt ions and solvent molecules. In addition to this inherent solvation, any sharp indentations on the macromolecule surface will contain solvent. All these "trapped" salt ions and solvent molecules are part of the macromolecule and will therefore travel in a solution with the same velocity as the latter.

The consequence is that the molecular dimensions computed by transport processes such as diffusion and viscosity, often called hydrodynamic processes, are much larger than the dimensions of the "real" macromolecule which can be obtained, for instance, from Xray diffraction and neutron scattering. Here, this can be seen very well from the behavior of $d_{\mathrm{h}}$. The average diameter of the $\mathrm{PSS}^{-}$skeleton cylinder is $1.5 \mathrm{~nm}$, that of the NaPSSskeleton, when no $\mathrm{H}_{2} \mathrm{O}$ is present, is $2.5 \mathrm{~nm}$. The hydrodynamic diameter, $d_{\mathrm{h}}$, is, on the other hand, much larger. Table I shows that $d_{\mathrm{h}}$ decreases from $13.1 \mathrm{~nm}$ for $I=0.005 \mathrm{M}$ to $3.5 \mathrm{~nm}$ at $I=2 \mathrm{M}$. The reason is clear. At small ionic strength, $I$, most of the sulfonate groups of PSS are dissociated. This leads to strong electric fields and a large number of $\mathrm{H}_{2} \mathrm{O}$ molecules is thus associated by hydrogenbonds to the polyion. At large $I$, on the other hand, most of the sulfonate groups are neutralized by $\mathrm{Na}^{+}$-ions. The attractive electrostatic forces are rather weak and the hydrodynamic volume $V_{\mathrm{h}}$ surrounding the $\mathrm{PSS}^{-}$-skeleton becomes small.

$V_{\mathrm{h}}$ is often devided in a number of solvation shells. Here, the thickness of such a solvation shell may be as large as the diameter of one solvated $\mathrm{Na}^{+}$-ion, where $d_{\mathrm{Na}^{+}} \approx 0.5 \mathrm{~nm}$. The average number of shells surrounding a PSSsegment of diameter $d_{\mathrm{h}}$ is thus given by $n_{\mathrm{s}}=\left[\left(d_{\mathrm{h}} / 2\right)-0.75 \mathrm{~nm}\right] / 0.5 \mathrm{~nm}$ with $R=0.75 \mathrm{~nm}$ being the radius of the $\mathrm{PSS}^{-}$-skeleton. For $I=2 \mathrm{M}, d_{\mathrm{h}}$ is $3.5 \mathrm{~nm}$, leading to $n_{\mathrm{s}}=2$. That is, at this high ionic strength, which lies near the $\theta$-state $\left(I_{\theta} \approx 3 \mathrm{M}\right)$, a $\mathrm{PSS}^{-}$-segment is surrounded by two solvation shells.
For a $\mathrm{PSS}^{-}$-ion, that is completely neutralized, $d_{\mathrm{h}}$ is $2.5 \mathrm{~nm}$ and $n_{\mathrm{s}}=1$. In our previous study, however, we have found that it is not possible to neutralize a PSS-ion completely. This implies that $n_{\mathrm{s}}$ must be larger than 1 also at the $\theta$-state. A value of $n_{\mathrm{s}}$ in the order of 2 for $I=2 \mathrm{M}$ is therefore reasonable. Note, the experimental error of $d_{\mathrm{h}}$ is between 10 and $15 \%$. The exact value of $n_{\mathrm{s}}$ may be therefore also smaller than 2 but in any case larger than 1. In conclusion, the behavior of $d_{\mathrm{h}}$ is consistent with the present stand of polyelectrolyte studies.

\section{The Effective Polyion Charge, $Z_{\text {eff }}$}

The number of charges per PSS-polyion, that are unscreened by $\mathrm{Na}^{+}$-ions, decreases with increasing distance from the polyion axis. That is, a hypothetical observer, who is placed at the distance $r$ from the polyion axis, will not feel the total number of charges but only a part $Z_{\text {eff }}$ of them.

$Z_{\text {eff }}$ is called the effective polyion charge. It can be measured by different experimental methods such as electrophoresis and electrophoretic light scattering. However, up to yet it is not clear to which distance $r$ from the polyion axis the $Z_{\text {eff }}$-values measured are referred. In addition, different methods yield different values for $Z_{\text {eff }}$ and a comparison between theory and experiment is therefore difficult.

Here, it seems to be most interesting to examine those values of $Z_{\text {eff }}$, which are seen by an observer, who is placed at the surface of a PSS-polyion cylinder with the diameter $d_{\mathrm{h}}$. That is, we ask what is the polyion charge fraction, $F\left(r_{h}\right)$, that is neutralized by counterions at a distance $r_{\mathrm{h}}=d_{\mathrm{h}} / 2$ from the polyion axis. $Z_{\text {eff }}$ is then given by $Z_{\text {eff }}=\left[1-F\left(r_{\mathrm{h}}\right)\right] \times$ $Z_{\text {total }}$, where $Z_{\text {total }}$ is the polyion charge when all sulfonate groups are ionized.

Note, $r_{\mathrm{h}}$ separates the hydrodynamic region, where the solvent components are parts of the polyion, from the region of the solution, where the solvent components are "free", i.e., 
where they travel with velocities different from those of the macromolecule. The relationship between $r_{\mathrm{h}}$ and $Z_{\text {eff }}$ is therefore of particular interest.

For simplicity we consider the polyion as a cylinder of radius $R$ bearing $N_{\mathrm{p}}$ fixed negative charges. The cylinder is entirely immensed in a larger cylinder of radius $R_{\infty}$ that contains solvent and a number of mobile small ions. The total charge inside the larger cylindrical volume is zero.

In order to obtain $F\left(r_{\mathrm{h}}\right)$ we have first to solve the Poisson-Boltzmann equation that is given by

$$
\begin{aligned}
V^{2} y(r)= & 4 \pi \xi b_{\mathrm{p}}\left[\left|Z_{1}\right|\left(n_{\mathrm{p}}\left(R_{\infty}\right)+{ }^{1} n_{\mathrm{S}}\left(R_{\infty}\right)\right)\right. \\
& \times \exp \left(+\left|Z_{1}\right| y(r)\right)-\left|Z_{2}\right|^{2} n_{\mathrm{S}}\left(R_{\infty}\right) \\
& \left.\times \exp \left(-\left|Z_{2}\right| y(r)\right)\right],
\end{aligned}
$$

where $y(r)=-e \Psi(r) / k_{\mathrm{B}} T$ is the reduced potential of the polyion. $b_{\mathrm{p}}$ is the axial distance per fixed charge on the polyion surface, $\xi$ is Manning's ${ }^{15}$ parameter, and $e$ the electronic charge. $n_{\mathrm{p}}\left(R_{\infty}\right)$ denotes the concentration of the counterions originating from the polyion ionization; ${ }^{1} n_{\mathrm{S}}\left(R_{\infty}\right)$ and ${ }^{2} n_{\mathrm{S}}\left(R_{\infty}\right)$ are the concentrations of the counter- and coions due to the added salt. $Z_{1}$ and $Z_{2}$ are the appertaining valences. The concentrations are given in terms of numbers per unit volume, and they are all related to $r=R_{\infty} \cdot n_{\mathrm{p}}\left(R_{\infty}\right)+{ }^{1} n_{\mathrm{S}}\left(R_{\infty}\right)$ and ${ }^{2} n_{\mathrm{S}}\left(R_{\infty}\right)$ are correlated with $y$ due to the condition of electroneutrality. It holds

$$
\begin{aligned}
& n_{\mathrm{p}}\left(R_{\infty}\right)+{ }^{1} n_{\mathrm{S}}\left(R_{\infty}\right) \\
&=\left(R_{\infty}^{2}-R^{2}\right)\left(N_{\mathrm{p}} \times c_{\mathrm{p}}+{ }^{1} N_{\mathrm{S}}\right) \\
&\left(2 \times \int_{R}^{R_{\infty}}\left(\exp \left|Z_{1}\right| y\right) r \mathrm{~d} r\right)
\end{aligned}
$$

and

$$
\begin{aligned}
{ }^{2} n_{\mathrm{S}}\left(R_{\infty}\right)= & \left(R_{\infty}^{2}-R^{2}\right) \\
& \times{ }^{2} N_{\mathrm{S}} /\left(2 \times \int_{R}^{R_{\infty}} \exp \left(-\left|Z_{2}\right| y\right) r \mathrm{~d} r\right)
\end{aligned}
$$

where ${ }^{1} N_{\mathrm{S}}$ and ${ }^{2} N_{\mathrm{S}}$ are the total numbers of positive and negative ions due to the added salt. $c_{p}$ is the number of polyions per unit volume. Here, we consider the case where $Z_{1}=-Z_{2}$ and $c_{\mathrm{p}}=0$. Thus, $R_{\infty} \gg 1 / \kappa$, i.e., $R_{\infty}=\infty$.

To calculate $y(r)$ we need two boundary conditions. These are given by applying Gauss' theorem at $r=R$ and $r=R_{\infty}$, that are

$$
\partial y(r) /\left.\partial r\right|_{R}=-2\left|Z_{\mathrm{p}}\right| \xi / R
$$

and

$$
\partial y(r) /\left.\partial r\right|_{R_{\infty}}=0
$$

$Z_{\mathrm{p}}$ is the valence of a polyion group, where $Z_{\mathrm{p}}=1$, here.

No one has ever obtained an analytical solution of the Poisson-Boltzmann equation when ${ }^{1} N_{\mathrm{S}}$ and ${ }^{2} N_{\mathrm{S}}$ are unequal to zero, i.e., in the presence of added salt; but a number of tables of numerically obtained solutions have been published. ${ }^{16-18}$ Here, we proceed as follows. Starting with an initial value of $y(R)$, a fourth-order Runge-Kutta method is applied to solve eq 7 numerically, using the ion numbers given by eq 8 and 9 and the boundary conditions given by eq 10 and 11 . If $y(r)$ and also $\partial y(r) / \partial r$ at $R_{\infty}=u \times \kappa^{-1}$ are not sufficiently small $\left(<10^{-3}\right), y(R)$ is varied systematically and the above procedure is repeated. $\kappa^{-1}$ is the Debye screening length, and $u$ is an arbitrary dimensionless constant in the order of 100 . The final results obtained for $y(r)$ are listed in Table II, where we have used the data sets $\xi=2.44, R=0.75 \mathrm{~nm}$ and $\xi=2.44, R=1.00 \mathrm{~nm}$ that are the most realistic one.

The fraction, $F(r)$, of the ionic charge that lies between the distance of closest approach $R$, and some arbitrary distance $r$ is given by

$$
F(r)=K \times \int_{R}^{r} p(r) r \mathrm{~d} r
$$

Here, $p(r)$ is the total charge density, and $K$ is a normalization constant. For the present case 


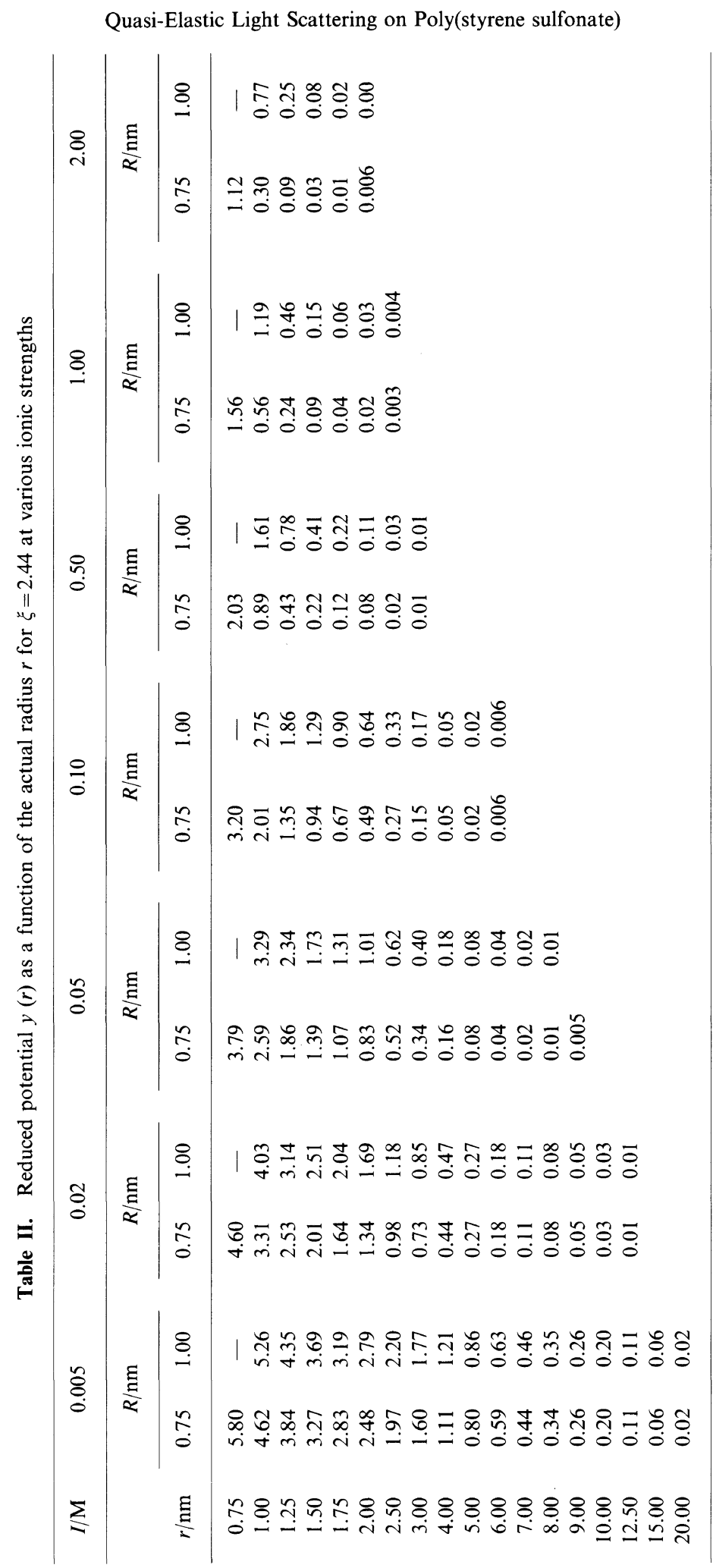


the above equation becomes

$$
\begin{aligned}
F(r)= & K \times e \times{ }^{1} N_{\mathrm{S}} \int_{R}^{r}[\exp (y(r)) \\
& -\exp (-y(r))] r \mathrm{~d} r .
\end{aligned}
$$

The results obtained for $F(r)$ with $R=1.0 \mathrm{~nm}$ at various $I$ are shown in Figure 3. $F(r)$ is zero at $r=1.0 \mathrm{~nm}$ and approaches to 1 as $r$ goes to infinity. If we set $r=d_{\mathrm{h}} / 2$, we get the $F\left(d_{\mathrm{h}} / 2\right)$-data given in Table III. Interestingly, $F(r)$ at $r=d_{\mathrm{h}} / 2$ does not depend on the ionic strength, $I$. For $R=0.75 \mathrm{~nm}$ the average of $F\left(d_{\mathrm{h}} / 2\right)$ is 0.96 and for $R=1.0 \mathrm{~nm}$ we have $\bar{F}\left(d_{\mathrm{h}} / 2\right)=0.94$. This yields an effective polyion charge, $Z_{\text {eff }}$, of 65.0 or 84.2 , respectively. It should be noted that the geometrical axial radius, $R$, of the ionized PSS-skeleton is

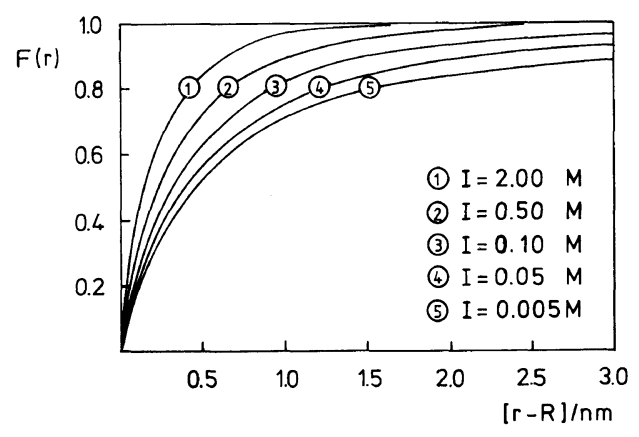

Figure 3. Plots of the fraction, $F(r)$, of the polyion charge that is neutralized by the small ions versus the distance $r$ from the NaPSS-axis. $\xi=2.44$ and $R=1.00 \mathrm{~nm}$.
$0.75 \mathrm{~nm}$, whereas $R$ is $1.25 \mathrm{~nm}$ for a NaPSSgroup. $R=1.0 \mathrm{~nm}$ presents the center of gravity of a bound $\mathrm{Na}^{+}$-ion. Thus, $Z_{\text {eff }}=84.2$ is the most likely value for $Z_{\text {eff }}$.

In a previous paper, ${ }^{1}$ we had used the dialysis equilibrium technique to determine the net number of polyion charges, $Z_{\text {net }}$, that are not neutralized by counterions within a volume $V_{\mathrm{p}}$ near the polyion surface. Now, we are able to calculate the average radius, $R_{\mathrm{PB}}$, of this volume and to compare the result with the radius, $R_{\mathrm{M}}$, suggested by Manning's ${ }^{15}$ condensation theory.

$R_{\mathrm{PB}}$ is easily obtained by eq 13 if we set $F\left(R_{\mathrm{PB}}\right)=1-\left(Z_{\text {net }} / Z_{\text {total }}\right)$. The results are listed in Table III. $R_{\mathrm{M}}$ is given by

$$
R_{\mathrm{M}}=29.4 \times \kappa \times\left[N_{\mathrm{A}} \times b_{\mathrm{p}} \times(1-1 / \xi) / c_{\mathrm{s}}\right]^{0.5},
$$

where $c_{\mathrm{S}}$ is the molar concentration of the added salt. Here, $\bar{R}_{\mathrm{M}}$ becomes $1.64 \mathrm{~nm}$ for all ionic strengths. The average value of $R_{\mathrm{PB}}$ is $1.54 \mathrm{~nm}$ for $R=1.0 \mathrm{~nm}$ and $1.33 \mathrm{~nm}$ for $R=0.75 \mathrm{~nm}$. This implies: (1) the experimental values of $Z_{\text {net }}$ are reasonable; (2) $R=1.0 \mathrm{~nm}$ is a good approximation for the radius, $R$, of the closest counterion-polyion approach; and (3) the condensation hypothesis of Manning does have a mathematical foundation in the

\begin{tabular}{|c|c|c|c|c|c|c|c|}
\hline \multirow{2}{*}{$I / \mathrm{M}$} & \multirow{2}{*}{$Z_{\text {net }}^{\mathrm{a}}$} & \multicolumn{3}{|c|}{$R=0.75 \mathrm{~nm}$} & \multicolumn{3}{|c|}{$R=1.00 \mathrm{~nm}$} \\
\hline & & $F\left(d_{h} / 2\right)$ & $Z_{\text {eff }}$ & $R_{\mathrm{PB}} / \mathrm{nm}$ & $F\left(d_{\mathrm{h}} / 2\right)$ & $Z_{\text {eff }}$ & $R_{\mathrm{PB}} / \mathrm{nm}$ \\
\hline 0.005 & 700 & 0.9597 & 63.3 & 1.3 & 0.9452 & 86.1 & 1.5 \\
\hline 0.01 & 596 & 0.9588 & 64.7 & 1.4 & 0.9443 & 87.5 & 1.6 \\
\hline 0.02 & 596 & 0.9602 & 62.5 & 1.4 & 0.9479 & 81.8 & 1.6 \\
\hline 0.05 & 565 & 0.9578 & 66.2 & 1.4 & 0.9452 & 86.1 & 1.6 \\
\hline 0.10 & 550 & 0.9597 & 63.3 & 1.3 & 0.9469 & 83.3 & 1.5 \\
\hline 0.20 & 518 & 0.9566 & 68.1 & 1.3 & 0.9461 & 84.7 & 1.5 \\
\hline 0.50 & 456 & 0.9609 & 61.3 & 1.3 & 0.9488 & 80.4 & 1.5 \\
\hline 1.00 & 345 & 0.9592 & 64.0 & 1.3 & 0.9462 & 84.5 & 1.5 \\
\hline 2.00 & 188 & 0.9602 & 62.5 & 1.3 & 0.9468 & 83.6 & 1.5 \\
\hline
\end{tabular}
Poisson-Boltzmann equation.

Table III. Charge parameters and radii of closest polyion-small ion approach

a See ref 1 . 


\section{The Hydrodynamic Radius}

The diffusive modes of polyions at different ionic strengths are determined by a complex set of interactions which involve solvent, polyion, counterion, and coion particles. In an attempt to elucidate these interactions Schurr ${ }^{5}$ derived for the translational diffusion coefficient, $D_{0}$, at $c_{\mathrm{p}}=0$ the expression

$$
\begin{aligned}
D_{0}= & k_{\mathrm{B}} T /\left[6 \pi \eta R_{\mathrm{h}}+\left(\left(Z_{\mathrm{eff}}^{2} e^{2} / 48 R_{\mathrm{h}}^{2} \varepsilon \varepsilon_{0} \kappa D_{\mathrm{S}}\right)\right.\right. \\
& \left.\left.\times\left(1-\left(\left(1+2 \kappa R_{\mathrm{h}}\right) \exp \left(-2 \kappa R_{\mathrm{h}}\right)\right)\right)\right)\right]
\end{aligned}
$$

The first term in the denominator is due to the solvent dissipation, while the second term is due to an asymmetric distribution of small ions about the polyion that provides an additional source of dissipation. $R_{\mathrm{h}}$ is the hydrodynamic radius, $Z_{\text {eff }}$ the effective polyion charge, $\varepsilon$ the bulk dielectric constant, $\kappa$ the Debye-Hückel screening length, and $D_{\mathrm{S}}$ the small-ion diffusion coefficient. All these parameters are given, except $R_{\mathrm{h}}$ that can be easily estimated by bisection. The results are given in Table IV, where also the radius of gyration, ${ }^{2}\left\langle S^{2}\right\rangle_{z}^{1 / 2}$, is listed. Due to the decrease of $Z_{\text {net }}$ and $d_{\mathrm{h}}$, respectively, both $\left\langle S^{2}\right\rangle_{z}^{1 / 2}$ and $R_{\mathrm{h}}$ decrease as the ionic strength is increased.

Of particular interest is the ratio $p=$ $\left\langle S^{2}\right\rangle_{z}^{1 / 2} / R_{\mathrm{h}}$. Since $\left\langle S^{2}\right\rangle_{z}^{1 / 2}$ is a geometrical average radius over the size of the polyion, while $R_{\mathrm{h}}$ is a hydrodynamic effective radius, $p$ depends no longer on the molecule dimension, but it depends on the structure and shape of the polyion. For monodisperse hard spheres ${ }^{19}$ of constant density one has $p=0.775$ and for linear flexible chains ${ }^{20}$ under $\theta$-conditions it holds $p=1.5$. The $p$-values of PSS reveal a change from $p=1.96$ at low $I$ to $p=1.63$ at high $I$.

The decrease in $p$ can be illustrated if the actual polyion is replaced by the model of an ellipsoid. For this purpose, we use a slightly modified formula of $p$, first derived by Perrin. ${ }^{21}$ For a prolate ellipsoid of revolution the result is

$$
\begin{aligned}
p= & 0.894 \times \ln \left[\left(1+\left(1-\vartheta^{2}\right)^{0.5}\right) / \vartheta\right] \\
& \times\left(\left(1+2 \vartheta^{2}\right) /\left(1-\vartheta^{2}\right)\right)^{0.5},
\end{aligned}
$$

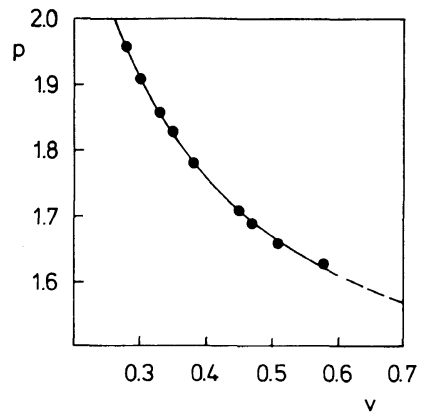

Figure 4. The ratio $p=\left\langle S^{2}\right\rangle_{z}^{1 / 2} / R_{\mathrm{h}}$ as a function of the ratio $\vartheta=b / a$.

Table IV. Structure parameters of PSS

\begin{tabular}{lcccccc}
\hline$I / \mathrm{M}$ & $\langle S\rangle_{\mathbf{z}}{ }^{\mathrm{a}} / \mathrm{nm}$ & $R_{\mathrm{h}} / \mathrm{nm}$ & $a / \mathrm{nm}$ & $b^{\mathrm{b}} / \mathrm{nm}$ & $\vartheta$ & $p$ \\
\hline 0.005 & 48 & 24.5 & 99.3 & 27.8 & 0.28 & 1.96 \\
0.01 & 45 & 23.6 & 92.7 & 27.8 & 0.30 & 1.91 \\
0.02 & 43 & 23.1 & 86.8 & 28.6 & 0.33 & 1.86 \\
0.05 & 41 & 22.4 & 81.8 & 28.6 & 0.35 & 1.83 \\
0.10 & 38 & 21.4 & 75.1 & 28.5 & 0.38 & 1.78 \\
0.20 & 36 & 21.1 & 67.9 & 30.6 & 0.45 & 1.71 \\
0.50 & 33 & 19.5 & 61.3 & 28.8 & 0.47 & 1.69 \\
1.00 & 30 & 18.1 & 54.5 & 27.8 & 0.51 & 1.66 \\
2.00 & 28 & 17.2 & 48.2 & 28.0 & 0.58 & 1.63 \\
\hline Error $\%$ & 10 & $2-5$ & - & - & - & $5-10$ \\
\hline
\end{tabular}

a See ref 2 .

b $\bar{b}=28.5 \pm 0.8 \mathrm{~nm}$. 
where $\vartheta=b / a$ is the ratio of the semiaxes $b$ and $a$. The major axis, $a$, is given by

$$
a=2 \times R_{\mathrm{h}} \times\left(1-\vartheta^{2}\right)^{-0.5} \ln \left[\left(1+\left(1-\vartheta^{2}\right)^{0.5}\right) / \vartheta\right]
$$

and the minor axis, $b$, by $b=\vartheta \times a$.

Values of $\vartheta, a$, and $b$ are listed in Table IV. $a$ decreases continuarly with $I$ while $b$ is independent on $I$. Figure 4 shows a plot of $p$ versus $\vartheta$. It seems that $\vartheta$ becomes 1 as $p$ approaches 1.5 , suggesting that in the $\theta$-state a PSS-polyion does have a spherical shape.

However, size changes are much easier to detect by measuring the rotational diffusion coefficient, $\theta$, than of $D_{0}$. It is therefore instructive to compare the $\theta$-value obtained by QELS with that calculated for an ellipsoid. Perrin ${ }^{21}$ has derived the relationship

$$
\theta_{\mathrm{E}}=\frac{3 k_{\mathrm{B}} T}{16 \pi \eta a^{3}}[2 \ln (2 a / b)-1],
$$

where the subscript " $E$ " stands for ellipsoid.

Values of $\theta_{\mathbf{E}}$ are listed in Table $\mathrm{I}$; they agree quite well with those $\theta$-values estimated for the model of the semiflexible rod. Additionally, it is seen that $L_{\mathrm{h}} \approx 2 \times a$. This implies: (1) the data of $L_{\mathrm{h}}$ and $a$ are reasonable; and (2) the results of the models used here are consistent, suggesting that a PSS-polyion can be described by a wormlike prolate ellipsoid.

The Concentration Dependence of $D_{\mathrm{app}}$

The dependence of the translational diffusion coefficient, $D_{\text {app }}$, at zero wave vector, $q$, on the polyion concentration is influenced by both thermodynamic and hydrodynamic interactions. It can be described by the relationship ${ }^{22}$

$$
\left.D_{\mathrm{app}}\right|_{q=0}=D_{0}\left[1+k_{\mathrm{D}, 2} \times c_{\mathrm{p}}+k_{\mathrm{D}, 3} \times c_{\mathbf{p}}^{2}+\cdots\right] .
$$

Here, $k_{\mathrm{D}, \mathrm{i}}$ are the iths hydrodynamic virial coefficients, and $c_{\mathrm{p}}$ the polyion concentration in $g$ per liter. For dilute solutions $k_{\mathrm{D}, 3}$ is zero, while $k_{\mathrm{D}, 2}$ is given by ${ }^{22}$

$$
k_{\mathrm{D}, 2}=2 A_{2} M_{w}-k_{\mathrm{f}}^{\mathrm{o}} \frac{N_{\mathrm{A}} \times V_{\mathrm{h}}}{M_{w}}-\bar{v}
$$

$A_{2}$ is the thermodynamic second virial coefficient, $M_{w}$ the molar mass, $N_{\mathrm{A}}$ Avogadro's number, $k_{\mathrm{f}}^{0}$ the friction coefficient at $\theta$ conditions, $V_{\mathrm{h}}$ the hydrodynamic volume of the particle, and $\bar{v}$ the partial specific volume of the polymer in solution. $\bar{v}$ is in the order of one and by orders of magnitude smaller than $2 A_{2} M_{w}$, it can be neglected in most cases. $k_{\mathrm{D}, 2}$ can be measured by means of QELS and $A_{2}$ by static light scattering. Both parameters are

Table V. Some other important properties of PSS

\begin{tabular}{ccccccc}
\hline$I / \mathrm{M}$ & $V_{\mathrm{h}} \times 10^{16} / \mathrm{cm}^{3}$ & $\begin{array}{c}A_{2} \times 10^{3 \mathrm{a}} / \\
\mathrm{mol} \mathrm{cm}^{3} \mathrm{~g}^{-2}\end{array}$ & $k_{\mathrm{D}, 2} / \mathrm{cm}^{3} \mathrm{~g}^{-1}$ & $k_{\mathrm{f}}^{\mathrm{o}}$ & $Z_{\mathrm{S}}^{\mathrm{b}}$ & $\begin{array}{c}\bar{A}_{2} \times 10^{-3} / \\
\mathrm{cm}^{3} \mathrm{~mol}^{-1}\end{array}$ \\
\hline 0.005 & 3.21 & 4.32 & 2572 & 0.82 & 94.9 & 95.62 \\
0.01 & 3.00 & 2.55 & 1380 & 0.78 & 98.3 & 64.26 \\
0.02 & 2.97 & 1.50 & 684 & 0.72 & 97.9 & 31.08 \\
0.05 & 2.80 & 0.77 & 260 & 0.58 & 95.4 & 10.05 \\
0.10 & 2.66 & 0.52 & 124 & 0.52 & 93.2 & 4.00 \\
0.20 & 2.55 & 0.40 & 68 & 0.48 & 97.6 & 3.04 \\
0.50 & 2.13 & 0.18 & 24 & 0.28 & 91.7 & 0.66 \\
1.00 & 1.76 & 0.10 & 12.4 & 0.19 & 93.2 & 0.40 \\
2.00 & 1.58 & 0.03 & 5.6 & 0.06 & 88.5 & 0.09 \\
\hline Error $\%$ & - & $3-5$ & 5 & - & - & - \\
\hline
\end{tabular}

a See ref 2 .

b $\bar{Z}_{\mathrm{s}}=94.5$. 
given here and listed in Table V. For $V_{\mathrm{h}}$ we use the volume of an ellipsoid that is

$$
V_{\mathrm{h}}=(4 \pi / 3) a \times b^{2}
$$

Thus, the only unknown parameter in eq 20 is that of $k_{\mathrm{f}}^{\circ}$.

There is no full agreement between the various theories ${ }^{22-24}$ on the value of $k_{\mathrm{f}}^{\mathrm{o}}$, but it seems generally be accepted that $k_{\mathrm{f}}^{\mathrm{o}}$ is a measure for the internal rigidity of a spherical particle. For instance, Pyun and Fixman ${ }^{6}$ found $k_{\mathrm{f}}^{\mathrm{o}}=7.2$ for the hard sphere and $k_{\mathrm{f}}^{\circ}=2.2$ for the soft sphere. The $k_{\mathrm{f}}^{0}$-values obtained for PSS are given in Table V. It is seen that $k_{\mathbf{f}}^{\circ}$ decreases with increasing ionic strength, $I$, indicating that the internal rigidity of a long "PSS-ellipsoid" is higher than that of a shorter one. It should be recalled that the minor axis, $b$, of the "PSS-ellipsoid" remains unchanged when $I$ is altered.

However, the dependence of $\left.D_{\text {app }}\right|_{q=0}$ on the polyion concentration, $c_{\mathrm{p}}$, can be also interpreted in terms of current polyelectrolyte theories $^{25-27}$, i.e., in terms of electrostatic interactions among the polyions and the surrounding small ions. Such a theoretical analysis was first proposed by Stephen, ${ }^{25}$ then by Schurr, $^{26}$ and latest by Magdelenat. ${ }^{27}$ Here, we employ the expression derived by Schurr because it is the most generally accepted one, and it is very close to that of Magdelenat. For a mono-monovalent salt Schurr obtained the relation

$$
\left.D_{\text {app }}\right|_{q=0}=1 / 2\left[D_{\mathrm{O}}(1-\Omega)+D_{\mathrm{S}}(1+\Omega)\right]
$$

with

$$
\Omega=\frac{D_{\mathrm{O}}-D_{\mathrm{S}}\left[1+\left(2 c_{\mathrm{S}} / c_{\mathrm{p}}\right) Z_{\mathrm{S}}^{-1}\right] Z_{\mathrm{S}}^{-1}}{D_{\mathrm{O}}+D_{\mathrm{S}}\left[1+\left(2 c_{\mathrm{S}} / c_{\mathrm{p}}\right) Z_{\mathrm{S}}^{-1}\right] Z_{\mathrm{S}}^{-1}},
$$

where $D_{\mathrm{S}}$ being the average diffusion coefficient of the counterions and coions. $Z_{\mathrm{S}}$ is Schurr's apparent (effective) polyion charge; $c_{\mathrm{S}}$ and $c_{\mathrm{p}}$ are the molar salt and polyion concentrations. In the dual limits $c_{\mathrm{S}} \gg Z_{\mathrm{S}} \times c_{\mathrm{p}}$, and $D_{\mathrm{S}} \gg D_{\mathrm{O}}$, eq 22 reduces to the familiar Donnan equilibrium form

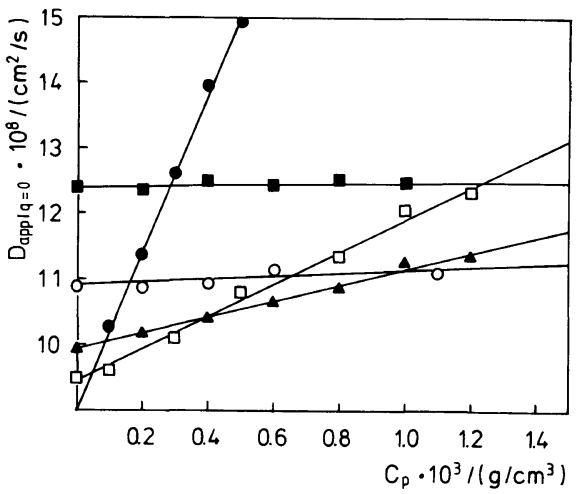

Figure 5. Plots of $\left.D_{\text {app }}\right|_{\mathbf{q}=0}$ versus $c_{\mathrm{p}}$ for various ionic strengths, $I$, at $T=20^{\circ} \mathrm{C} . I=0.01(\mathbf{O}), 0.05(\square), 0.1(\mathbf{\Delta})$, $0.5(\bigcirc)$, and $2 \mathrm{M}(\boldsymbol{\square})$.

$$
\left.D_{\mathrm{app}}\right|_{q=0}=D_{\mathrm{O}}\left[1+\left(Z_{\mathrm{S}}^{2} / 2 c_{\mathrm{S}}\right) c_{\mathrm{P}}\right],
$$

where $\left.D_{\text {app }}\right|_{q=0}$ no longer depends on the small ion diffusion coefficient, $D_{\mathrm{S}}$. Figure 5 shows that $\left.D_{\text {app }}\right|_{q=0}$ is a linear function of $c_{\mathrm{p}}$ for all $c_{\mathrm{S}}$. In particular, $D_{\mathrm{O}}$ increases and $Z_{\mathrm{S}}^{2} / 2 c_{\mathrm{S}}$ decreases as $c_{\mathrm{S}}$ is increased. A peculiar feature of Figure 5 is the presence of common values for $\left.D_{\text {app }}\right|_{q=0}$ at certain concentrations $c_{\mathrm{p}}^{\mathrm{s}}$, causing two different trends in $\left.D_{\text {app }}\right|_{q=0}$ with regard to $c_{\mathrm{S}}$. At a fixed value of $c_{\mathrm{p}}$ the diffusion coefficient $\left.D_{\text {app }}\right|_{q=0}$ decreases with $c_{\mathrm{S}}$ as long as $c_{\mathrm{p}}<c_{\mathrm{p}}^{\mathrm{S}}$, while for $c_{\mathrm{p}} \geq c_{\mathrm{p}}^{\mathrm{s}}$ the coefficient $\left.D_{\text {app }}\right|_{q=0}$ increases. This is the result of two facts: (1) a PSS-chain expands when the ionic strength is decreased, and (2) the coupled small ion-polyion interactions loose partly their importance when I is increased.

Table V shows that Schurr's polyion charge, $Z_{\mathrm{s}}$, does not depend on the ionic strength, $I$, as expected. However, $\bar{Z}_{\mathrm{S}}=94.5$ is a little larger than $\bar{Z}_{\text {eff }}=84.2$ obtained by solving the Poisson-Boltzmann equation. This discrepancy may have two reasons: (1) Schurr's eq 22 is a zero-order approximation, i.e., strictly valid at infinite dilution, that does not account the electrostatic coupling of polyionsmall ion and polyion-polyion interactions, and (2) the Poisson-Boltzmann approach is also defective with regard to the fact that nonelectrostatic interactions among the small ions are neglected. 
Recently, Schmitz ${ }^{28}$ has proposed to introduce a virial coefficient $\bar{A}_{2}$ into eq 23 that accounts direct polyion-polyion interactions. He therefore wrote

$$
\left.D_{\text {app }}\right|_{q=0}=D_{\mathrm{O}}\left[1+\left[\left(Z_{\text {eff }}^{2} / 2 c_{\mathrm{S}}\right)+2 \bar{A}_{2}\right] c_{\mathrm{p}}\right],
$$

where he additionally replaced $Z_{\mathrm{s}}$ by $Z_{\text {eff }}$. The results obtained for $\bar{A}_{2}$ by using this corrected equation are summarized in column (7) of Table V. It is seen that $\bar{A}_{2}$ is large at low $I$ and very small at high $I$, suggesting that the polyion-polyion interactions looses their significance as $I$ is increased. This finding is reasonable, however, eq 24 is not very useful as long as $\bar{A}_{2}$ has no fundamental physical base. In order to determine $Z_{\text {eff }}$ we need a mathematical expression for $\bar{A}_{2}$ that only depends on $Z_{\text {eff }}$ and parameters given by the experimentation. Thus, there remains a problem that we hope to solve in the immediate future.

\section{CONCLUSIONS}

Our method of analysis discussed above seems promising in elucidating the dynamics of wormlike polyelectrolytes in the dilute regime at different ionic strengths. Important problems which will be studied in the future are (1) to give eq 4 a fundamental physical base, or to derive an alternative; (2) to establish the size of the parameter, $d_{h}$, by other experimental methods in order to ensure that $Z_{\text {eff }}$ is independent of $I$; (3) to prove the Poisson-Boltzmann equation in some details, that is not an exact result of statistical mechanics; (4) to derive a hydrodynamic theory of $D_{\text {app }}$ that describes in a general manner the coupling of polyion-polyion and polyionsmall ions interactions; and (5) to establish the method of analysis used here for more diversified polyion-small ion systems over a wide range of boundary conditions. All of these problems are now under consideration.

Acknowledgements. I thank Prof. Dr. M.
D. Lechner for providing laboratory facilities and for helpful discussions. The research was supported by the "Fonds der Chemischen Industrie".

\section{REFERENCES}

1. E. Nordmeier and W. Dauwe, Polym. J., 23, 1297 (1991).

2. E. Nordmeier and W. Dauwe, Polym. J., 24, 229 (1992).

3. E. Nordmeier, Polym. J., 25, 1 (1993).

4. T. Maeda and S. Fujime, Macromolecules, 17, 2381 (1984).

5. J. M. Schurr, J. Chem. Phys., 45, 119 (1980).

6. C. W. Pyun and M. J. Fixman, J. Chem. Phys., 41, 937 (1964).

7. S. C. Lin, W. I. Lee, and J. M. Schurr, Biopolymers, 17, 1041 (1978).

8. E. Nordmeier and M. D. Lechner, Polym. J., 21, 623 (1989).

9. R. Pecora, "Dynamic Light Scattering," Plenum Press, New York, N.Y., 1985.

10. S. Fujime and T. Maeda, Macromolecules, 18, 191 (1985).

11. T. Maeda and S. Fujime, Macromolecules, 17, 1157 (1984).

12. T. Maeda and S. Fujime, Macromolecules, 18, 2430 (1985).

13. J. E. Hearst and W. H. Stockmayer, J. Chem. Phys., 37, 1425 (1962).

14. S. Fujime, M. Takasaki, and T. Maeda, Macromolecules, 20, 1292 (1987).

15. G. S. Manning, Q. Rev. Biophys., 11, 179 (1978).

16. D. Stigter, J. Colloid Interface Sci., 53, 296 (1975).

17. J. A. Schellman and D. Stigter, Biopolymers, 16, 1415 (1977).

18. M. Le Bret and B. H. Zimm, Biopolymers, 23, 287 (1984).

19. W. Burchard, Macromolecules, 11, 455 (1978).

20. W. Burchard and G. D. Patterson, "Advances in Polymer Science, 48," Springer Press, New York, N.Y., 1983.

21. F. Perrin, J. Phys. Radium, 5, 497 (1934).

22. H. Yamakawa, "Modern Theory of Polymer Solutions," Harper and Row, New York, N.Y., 1971.

23. S. Imai, J. Chem. Phys., 50, 2116 (1969).

24. P. Flory and W. R. Krigbaum, J. Chem. Phys., 18, 1086 (1950).

25. M. J. Stephen, J. Chem. Phys., 55, 3878 (1971).

26. J. M. Schurr, CRC Crit. Rev. Biochem., 4, 371 (1977).

27. P. Trivant and H. Magdelenat, Biopolymers, 22, 643 (1983).

28. K. Schmitz, Macromolecules, 16, 1550 (1983). 